\title{
Kopfschmerzbehandlung mit Akupunktur aus Sicht der TCM
}

\author{
Stefan Kirchhoff
}

\author{
Kopfschmerzen können mit Akupunktur auf vielfältige Weise behandelt werden. So besteht \\ die einfache und pragmatische Möglichkeit, nach der Kopfschmerzlokalisation entspre- \\ chend der Leitbahnversorgung zu behandeln. Komplexer und individueller ist die zusätzliche \\ Differenzierung nach der chinesischen Syndromlehre. Die Kombination beider Konzepte \\ führt zu effektiven Behandlungsansätzen für sowohl akute als auch chronische Fälle.
}

Kopfschmerzen gehören neben Knie- und Rückenschmerzen zu den häufigsten Akupunkturindikationen in der täglichen Praxis. Zusätzlich zur unabdingbaren schulmedizinischen westlichen Abklärung erweist sich eine klassisch-chinesische Differenzialdiagnostik für die Punktauswahl als hilfreich. Standard-Punktkombinationen können in einigen Fällen zum Erfolg führen, versagen aber oft bei Patienten, denen bei einer entsprechenden weiteren Differenzierung ihrer Kopfschmerzen durchaus noch geholfen werden kann. Der folgenden Artikel soll eine Hilfestellung sowohl für das grundlegende Verständnis als auch für die Diagnostik und Therapie von Kopfschmerzen aus Sicht der Traditionellen Chinesischen Medizin (TCM) in der Praxis sein.

\section{Allgemeine Grundlagen}

\section{Bedeutung und Ursachen von Schmerz in der TCM}

In der TCM gilt, dass dort, wo Schmerz auftritt, eine Stagnation der Zirkulation von Qi und/oder Blut-Xue vorliegt. Das trifft auch für Kopfschmerzen zu. Eine Behandlung von Schmerzen beinhaltet somit als ein vorrangiges Therapieprinzip die Wiederherstellung der freien Zirkulation von Qi und Blut-Xue mit Akupunktur, aber auch anderen Verfahren der TCM wie Moxa, Schröpfen, Mikroaderlass, Gua Sha (Schaben), Tuina, Qi Gong und Phytotherapie.

Die symptomatische Auflösung von Stagnationen und Stasen führt oft zu kurzfristigen Verbesserungen, beseitigt jedoch häufig nicht die Ursache, die zur Stagnation führt. Die Folge sind rezidivierende Verläufe. Für eine kausale Therapie der zugrunde liegenden Disharmonien ist ein Verständnis der Schmerzursachen in der TCM hilfreich:

- Physische Traumata, z. B. Schädel-Hirn- oder HWSSchleudertraumata, führen zu einer abrupten
Unterbrechung der Zirkulation von Qi und insbesondere von Blut-Xue.

- Auch das Eindringen von äußeren klimatischen pathogenen Faktoren (z. B. Wind, Kälte, Feuchtigkeit) in die tendinomuskulären Leitbahnen, die LuoGefäße oder Hauptleitbahnen kann zu Stagnationen führen. Führend ist dabei meist Wind, der die Oberfläche öffnet, damit andere Faktoren wie u. a. Kälte oder Feuchtigkeit eindringen können. Wir kennen diesen Mechanismus bei Kopfschmerzen im Zusammenhang mit Erkältungskrankheiten oder nach Einwirken von starker Zugluft.

- Eine Stagnation im Kopf und somit Schmerz kann aber auch entstehen, wenn der Kopfbereich aus einem Mangel heraus zu wenig mit Qi oder Blut-Xue versorgt wird. In diesem Fall entsteht die Stagnation, weil die Antriebskraft für die Aufrechterhaltung der Zirkulation zu gering ist. Dann finden sich oft Kopfschmerzen im Zusammenhang mit Erschöpfung. Auch akute Kopfschmerzen bei Flüssigkeitsmangel sind dafür ein Beispiel.

- Ein häufiges Phänomen bei chronischen Schmerzen ist die Kombination von einem inneren Mangel-Syndrom mit gleichzeitiger Fülle. Einerseits erleichtert eine vorbestehende Schwäche von Blut-Xue, Qi oder anderen „Körpersubstanzen“ das Eindringen von äußeren pathogenen Faktoren durch eine geschwächte Abwehr, andererseits kann das Persistieren von äußeren pathogenen Faktoren langfristig zu einer Schwächung der Zang Fu führen. Zudem gibt es Fülle-Zustände, die sich aus Mangel-Syndromen entwickeln können, wie z. B. Nässe und Schleim als Folge eines Milz-Qi-Mangels oder aufsteigendes Yang aufgrund eines Yin-Mangels.

- Übermäßige oder inadäquate Emotionen gelten als innere Fülle und können die Zirkulation blockieren. Das gilt insbesondere für Wut, Ärger, Frustration und alle unterdrückten, nicht ausgelebten Emotionen. 


\section{Differenzierung von Kopfschmerzen}

Aus den genannten Mechanismen wird deutlich, dass man für eine exakte Diagnose von Kopfschmerzen mehrere Aspekte in Form grundlegender Konzepte der TCM berücksichtigen kann und sollte. Kopfschmerzen erklären sich aus dem Zusammenspiel

- der Einflüsse des Leitbahnsystems,

- der Funktion der inneren Zang-Fu-Organe inklusive der ihnen zugehörigen Emotionen,

- dem Mangel oder der Stagnation der Körpersubstanzen und

- dem eventuellen Vorliegen von äußeren pathogenen Faktoren.

Welche Fragen sollte man sich bezüglich eines Patienten mit Kopfschmerzen stellen?

1. Welche Leitbahn versorgt das betroffene Kopfschmerzareal? Das ist die wichtigste Frage, da die Effekte der Akupunktur über die Leitbahnen vermittelt werden. Sie führt zur Auswahl der korrekten leitbahnbezogenen Fernpunkte.

2. Handelt es sich um ein äußeres Disharmoniemuster? Eine äußere Störung entsteht oft akut durch äußere pathogene Faktoren oder muskuläre Fehlbelastungen. Bei der Therapie reichen leitbahnorientierte Punktkombinationen oft aus. Tritt der akute Kopfschmerz z. B. nach starkem Wind-Einfluss oder zusammen mit einem Infekt auf? Reagiert der Patient bei Rezidiven immer wieder auf klimatische Einflüsse? Dann könnten äußere pathogene Faktoren beteiligt sein. Neben Wind-Kälte, -Hitze oder -Feuchtigkeit ausleitenden Punkten können auch Verfahren wie Gua Sha oder Schröpfen ergänzend eingesetzt werden.

3. Handelt es sich um ein inneres Disharmoniemuster? Dann müssen die Zang-Fu-Organe mit den Körpersubstanzen in die Diagnostik mit einbezogen werden. Es kann sich z. B. um eine hormonabhängige Migräne handeln, aber auch durch Emotionen getriggerte Kopfschmerzen gehören dazu. Die am häufigsten betroffenen Zang Fu im Zusammenhang mit Kopfschmerzen sind Leber, Gallenblase, Niere und Magen. Diese differenziert man anhand der vorliegenden Begleitsymptome oder der deutlichen Zungenbefunde. Die leitbahnorientierten Punkte werden dann um Punkte ergänzt, die das erkannte Zang-Fu-Syndrom behandeln.

4. Liegt ein Fülle- oder Leere-Muster vor? Die Beantwortung dieser Frage führt zur korrekten Nadeltechnik und adjuvanten Therapieverfahren. Bei Mangel wird tonisierend/auffüllend (Bu Fa) genadelt und beim Ausschluss von Hitze kann auch Moxa zur Anwendung kommen. Bei Fülle behandelt man mit sedierender/zerstreuender/ableitender Nadeltechnik (Xie Fa). Ergänzend können Schröpfen, Mikroaderlass oder Gua Sha eingesetzt werden. Cave: Die häufig vorgenommene pauschale Unterscheidung in „chronisch = Leere“ und „akut = Fülle“ reicht nicht aus und ist häufig nicht korrekt.

\section{Therapiestrategien}

Für die praktische Therapie lassen sich daraus 3 Hauptstrategien für die Kopfschmerzbehandlung ableiten:

1. betroffene Leitbahnen durchgängig machen

2. äußere pathogene Faktoren, falls vorhanden, ausleiten

3. eventuell zugrunde liegende innere Zang-Fu-Syndrome behandeln

Es ist naheliegend, dass Akupunkteure den Fokus der Behandlung auf die Leitbahnbehandlung legen. Die Leitbahnen sind die auf eine Akupunktur zuerst reagierenden Funktionseinheiten. Erst dann werden pathogene Faktoren ausgeleitet oder die Zang Fu reguliert.

\section{Leitbahnversorgung des Kopfs}

In der TCM wird der Kopf mit dem Himmel verglichen. Er repräsentiert somit das Yang mit seinen Attributen wie Oben, Außen und Wärme. Man bezeichnet ihn auch als den Palast des Yang. Er besitzt so viel Yang, dass man den Kopf im Allgemeinen nachts, im Gegensatz zum restlichen Körper, nicht zudecken muss, um ihn vor Kälte zu schützen. Er ist der Ort des größten Energiepotenzials in der Leitbahnzirkulation. Keine andere Körperregion hat eine so vielfältige und dichte Leitbahnversorgung wie der Kopf.

Alle Yang-Leitbahnen beginnen oder enden am Kopf. Von den Yin-Leitbahnen hat nur die Leber-Leitbahn einen direkten Ast zum Kopf bis hinauf zu LG 20 (Du 20). Weniger geläufig ist, dass auch die anderen Yin-Leitbahnen über die Divergierenden Leitbahnen (Jing Bie) Einfluss auf den Kopf haben. Dies geschieht über die oberen Vereinigungspunkte der jing Bie. So beeinflusst z. B. die Nieren-Leitbahn über BI 10 den Kopf und die Milz-Leitbahn über Ma 9. Bis auf Dai Mai ziehen auch alle anderen der 8 außerordentlichen Leitbahnen zu Kopf und Gesicht.

LEITBAHNEN DES KOPFS

- alle Yang-Leitbahnen: Dünndarm, 3-Erwärmer, Dickdarm, Blase, Gallenblase, Magen

- Leber-Leitbahn

- weitere Yin-Leitbahnen über die Divergierenden Leitbahnen

- alle außerordentliche Leitbahnen außer Dai Mai

\section{Grundtypen des Kopfschmerzes}

Die einfachste Art, Kopfschmerzen mit Akupunktur zu behandeln, ist die Differenzierung nach den 4 
Grundtypen des Kopfschmerzes. Dabei wird der Kopfschmerz nach seiner Lokalisation klassifiziert ( Abb.1, - Tab.1). Diese Unterteilung ist leitbahnorientiert und wird nach den Liu Jing, den 6 großen Hauptleitbahnen, auch als Oben-Unten-Kopplungen oder Leitbahnachsen bekannt, vorgenommen ( $\triangleright$ Tab. 2). Allerdings werden nur 4 der 6 Liu Jing zur Kopfschmerzdifferenzierung verwendet, da nur die Hauptleitbahnen dieser Achsen einen direkten Bezug zum Kopf haben.

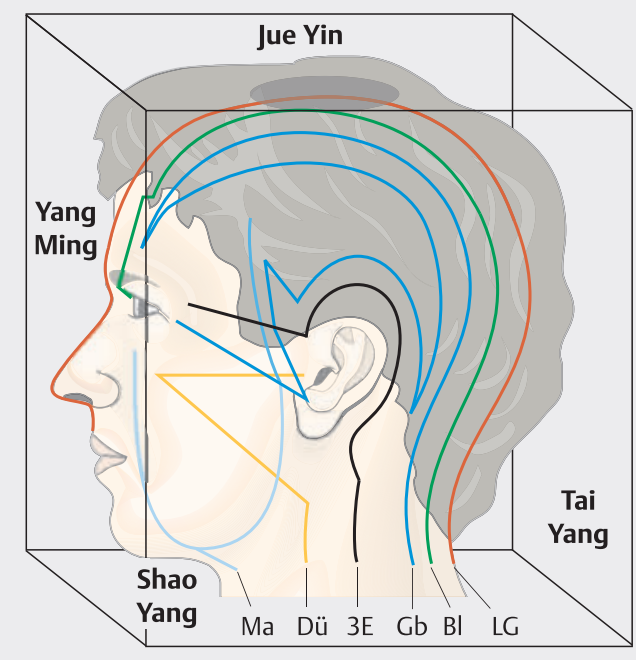

- Abb. 1 Kopfschmerzareale nach Liu jing. Quelle: Thieme Gruppe

-Tab. 1 Kopfschmerzareale nach Liu Jing.

\begin{tabular}{|l|l|}
\hline betroffene Leitbahn & Schmerzlokalisation \\
\hline Tai Yang & okzipital \\
\hline Shao Yang & temporal \\
\hline Yang Ming & frontal \\
\hline Jue Yin & auf dem Vertex \\
\hline
\end{tabular}

\section{Tai-Yang-Kopfschmerz}

Der Tai-Yang-Kopfschmerz korrespondiert mit der Blasen- und Dünndarm-Leitbahn. Er manifestiert sich primär im Hinterhauptsbereich und im dorsalen Nacken. Seltener strahlt er, dem Leitbahnverlauf folgend, bis zum Vertex oder sogar in die Stirn aus.

Wichtige Akupunkturpunkte zur Behandlung des TaiYang-Kopfschmerzes sind ( $>$ Abb. 2):

- Lokal-/Nahpunkte: Bl 10 (Tian Zhu), BI 9 (Yu Zhen), LG 16 (Feng Fu), Gb 20 (Feng Chi)

- Fernpunkte: Bl 60 (Kun Lun), Lu 7 (Lie Que), Dü 3 (Hou Xi)

BI 10 und BI 9 als Lokalpunkte sind selbsterklärend. Auch LG 16 liegt direkt unter der Protuberantia occipitalis und somit im definierten Areal. Als Punkt des Du Mai (Lenkergefäß) ist er über dessen Öffnungspunkt Dü 3 und den Anknüpfungspunkt Bl 62 funktional eng mit Tai Yang verbunden. Gb 20 ist ein wichtiger Punkt für die gesamte Nackenregion und leitet äußere pathogene Faktoren, v. a. Wind, aus der Kopfregion aus.

Allen genannten Fernpunkten ist gemeinsam, dass sie an den Händen, Füßen oder den Hand- bzw. Fußgelenken liegen. Hände und Füße sind die korrespondierenden Areale für den Kopf. Die Hand- bzw. Fußgelenke repräsentieren die Nackenregion und die HWS. Mit der Kombination BI60 und Dü 3 spricht man die gesamte Tai-Yang-Achse mit Betonung von Kopf und Nacken an. Bl 60 ist der 4. Antike Jing-Punkt und hat eine starke Qibewegende Wirkung auf die Blasen-Leitbahn. Außerdem ist er ein wichtiger Fernpunkt für Nacken- und Okziput. Dü 3 ist der Yuan-Quellpunkt der DünndarmLeitbahn und benutzt somit die Dynamik des Yuan Qi, um Stagnation zu lösen. Er ist auch der Öffnungspunkt des Du Mai und stellt darüber eine sinnvolle Verbindung zu LG 16 her. Somit gilt er als wichtiger Fernpunkt für Nacken, Okziput und Rücken. Auch Lu 7 liegt nahe des Handgelenks und ist als einer der Kommandopunkte (regionale Fernpunkte) nach Gao Wu für Nacken und Okziput indiziert. In Akupunktursystemen, die mit der Leitbahnbalancierung arbeiten, kann die Lungen-

$>$ Tab. 2 Liu Jing - die 6 Leitbahnachsen.

\begin{tabular}{|l|l|l|}
\hline Achse & Arm & Bein \\
\hline Tai Yang & Dünndarm & Blase \\
\hline Shao Yang & 3-Erwärmer & Gallenblase \\
\hline Yang Ming & Dickdarm & Magen \\
\hline Tai Yin & Lunge & Milz \\
\hline Shao Yin & Herz & Niere \\
\hline Jue Yin & Perikard & Leber \\
\hline
\end{tabular}




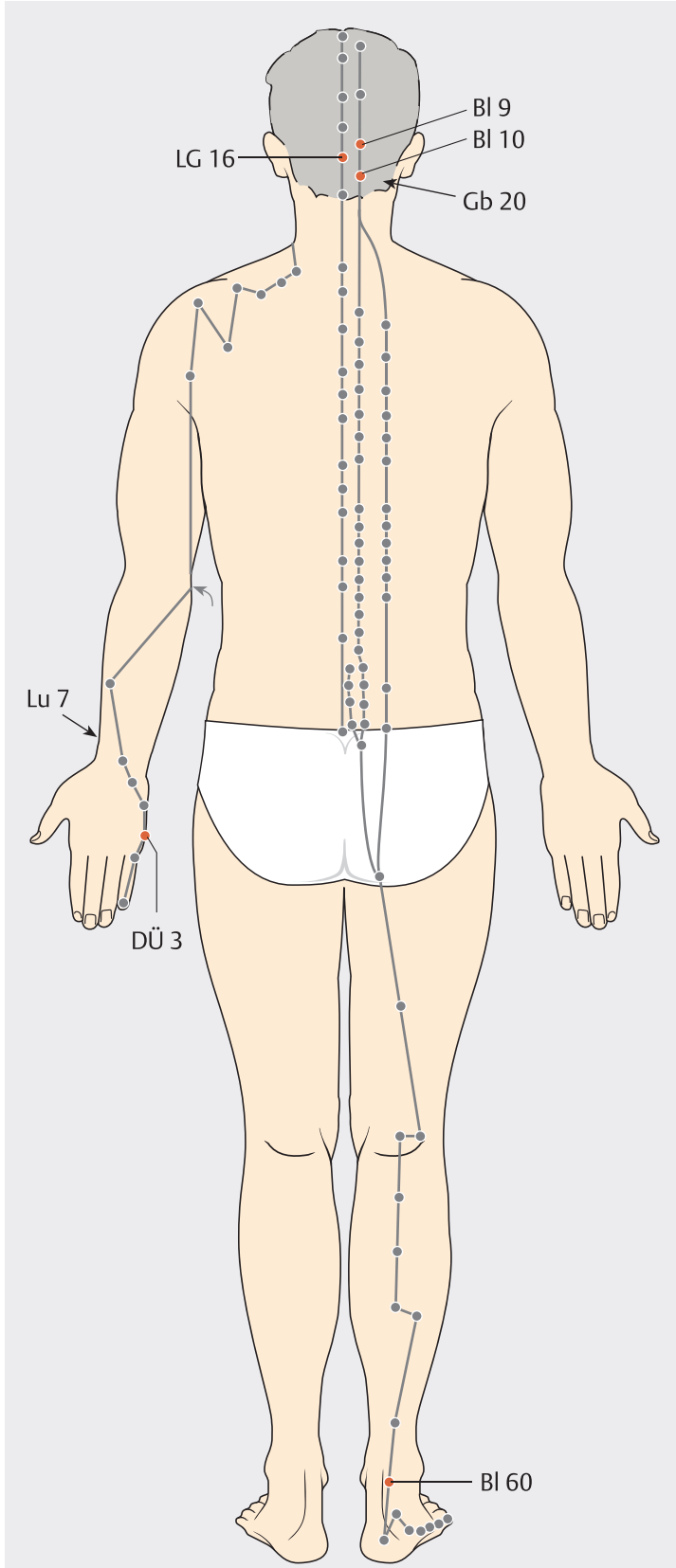

Abb. 2 Punktauswahl beim Tai-Yang-Kopfschmerz. Quelle: Thieme Gruppe

Leitbahn die Blasen-Leitbahn harmonisieren, da beide in der Organuhr in Opposition zueinander stehen.

\section{Shao-Yang-Kopfschmerz}

Der Shao-Yang-Kopfschmerz korrespondiert mit der Gallenblasen- und 3-Erwärmer-Leitbahn. Er manifestiert sich typischerweise als Halbseiten- oder Schläfenkopfschmerz. Meist ist er einseitig lokalisiert, aber auch ein beidseitiges Auftreten ist möglich. Der Shao-Yang-Kopfschmerz findet sich in der Praxis häufig, da auch die klassische Migräne hier einzuordnen ist. Die Schmerzen können in die Ohren oder die Augen ausstrahlen.

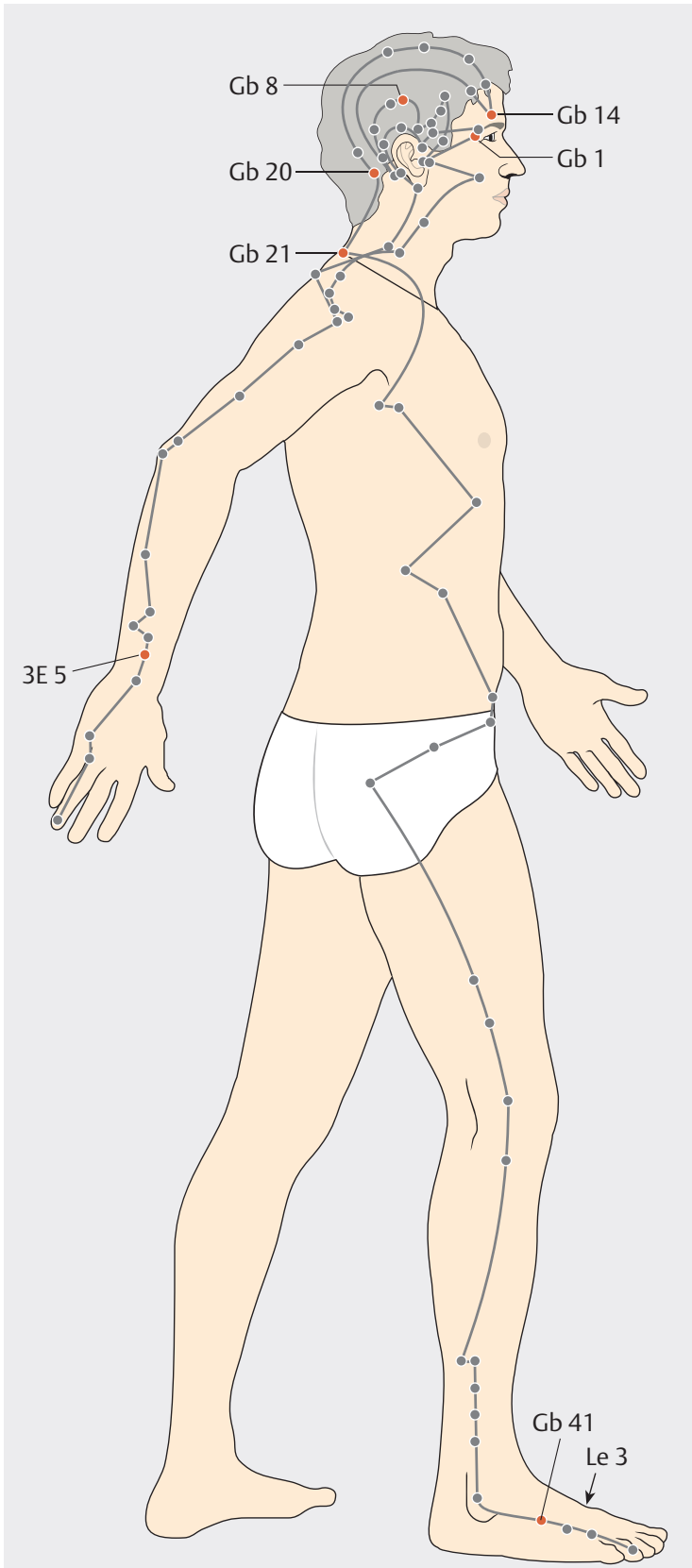

Abb. 3 Punktauswahl beim Shao-Yang-Kopfschmerz. Quelle: Thieme Gruppe

Wichtige Akupunkturpunkte zur Behandlung des ShaoYang-Kopfschmerzes sind ( $\mathbf{A}$ Ab. $\mathbf{3}$ ):

- Lokal-/Nahpunkte: Gb 20 (Fengchi), Gb 8 (Shuai Gu), Gb 1 (Tong Zi Liao), Extrapunkt Tai Yang (Ex-KH 5), Ma 8 (Tou Wei), Gb 14 (Yang Bai), Gb 21 (Jian Jing)

- Fernpunkte: 3 E 5 (Wai Guan), Gb 41 (Zu Lin Qi), Le 3 (Tai Chong)

Die genannten Lokal-/Nahpunkte sollten nicht alle in einer Sitzung genadelt werden. Man trifft eine Auswahl und wechselt evtl. bei der nächsten Sitzung die Punkte. 
Gb20 ist einer der wichtigsten Kopfschmerzpunkte überhaupt. Er klärt die Nacken- und Kopfregion. Er ist der Punkt der Wahl, egal ob es sich um muskulär oder emotional bedingte Spannungskopfschmerzen, Erkältungskopfschmerzen oder Migräne handelt. Er löst Stagnationen, leitet Wind aus und lässt übermäßig aufsteigendes Yang von Leber und Gallenblase über seine Verbindung zur Außerordentlichen Leitbahn Yang Wei Mai nach unten abfließen. Dies erklärt sein breites Wirkspektrum und auch, dass er sowohl bei inneren als auch bei äußeren Syndromen Verwendung findet.

Gb 8 ist ein wichtiger Lokalpunkt und wird auch beim Katerkopfschmerz genadelt. Gb 1 ist insofern ein besonderer Punkt, da er neben seiner lokalen Wirkung der obere Vereinigungspunkt der Divergierenden Leitbahnen von Gallenblase und Leber ist. Wir werden später sehen, dass die Ursachen für Shao-Yang-Kopfschmerzen oft in Dishamoniemustern der Leber zu finden sind. Mit Gb 1 nadelt man also gleichzeitig die Shao-Yang-Achse und die Leber-Leitbahn. Gb 21 ist besonders dann indiziert, wenn die Schulterregion sehr verspannt ist. Bei starken Verspannungen kann noch Gb 34 als Hui-EinflussreicherPunkt für Muskeln und Sehnen genadelt werden.

Die meisten Fernpunkte liegen an der Hand oder am Fuß. $3 E 5$ als Ausnahme ist der Öffnungspunkt des Yang Wei Mai, insbesondere in der Kombination mit Gb41. Yang Wei Mai ist eng mit der Shao-Yang-Achse verbunden und nutzt viele Gallenblasen-Punkte am Kopf (u.a. Gb 20) mit ihr gemeinsam. $3 E 5$ ist ein wichtiger Punkt für seitlichen Kopfschmerz, macht die Leitbahn durchgängig und vertreibt auch äußeren Wind. Gb41 ist in diesem Kontext der Anknüpfungspunkt des Yang Wei Mai und wird oft in Kombination mit $3 E 5$ akupunktiert. Dabei nadelt man erst $3 E 5$ einseitig bei Frauen links und bei Männern auf der rechten Seite. Danach sticht man Gb 41 kontralateral. Die gemeinsame Nadelung von 3E 5, Gb41 und Gb20 stellt eine elegante und wirksame Punktkombination dar. Sowohl 3E 5 als auch Gb 41 können auch alleine für sich genadelt werden, ohne den Yang Wei Mai zu öffnen. Le 3 ist kein Punkt der ShaoYang-Achse und weist deutlich auf eine tiefere und häufige Ursache des temporalen Kopfschmerzes im LeberFunktionskreis hin. Le 3 ist der Yuan-Quell-Punkt, der 3. Antike Shu-Bachpunkt und der Erde-Punkt der LeberLeitbahn.

Die 3. Antiken Shu-Punkte behandeln direkt die ZangOrgane und sind bei intermittierend auftretenden Symptomen indiziert. Le 3 kann also bei allen Leber-Syndromen zum Einsatz kommen, v.a., wenn sie mit rezidivierend auftretenden Kopfschmerzen zu tun haben. Als Yuan-Punkt hat er eine zusätzliche, das Leber-Qi bewegende Qualität. Aus seiner Eigenschaft als ErdePunkt erklärt sich die balancierende und nährende Wirkung, sodass er neben der Leber-Qi-Stagnation auch

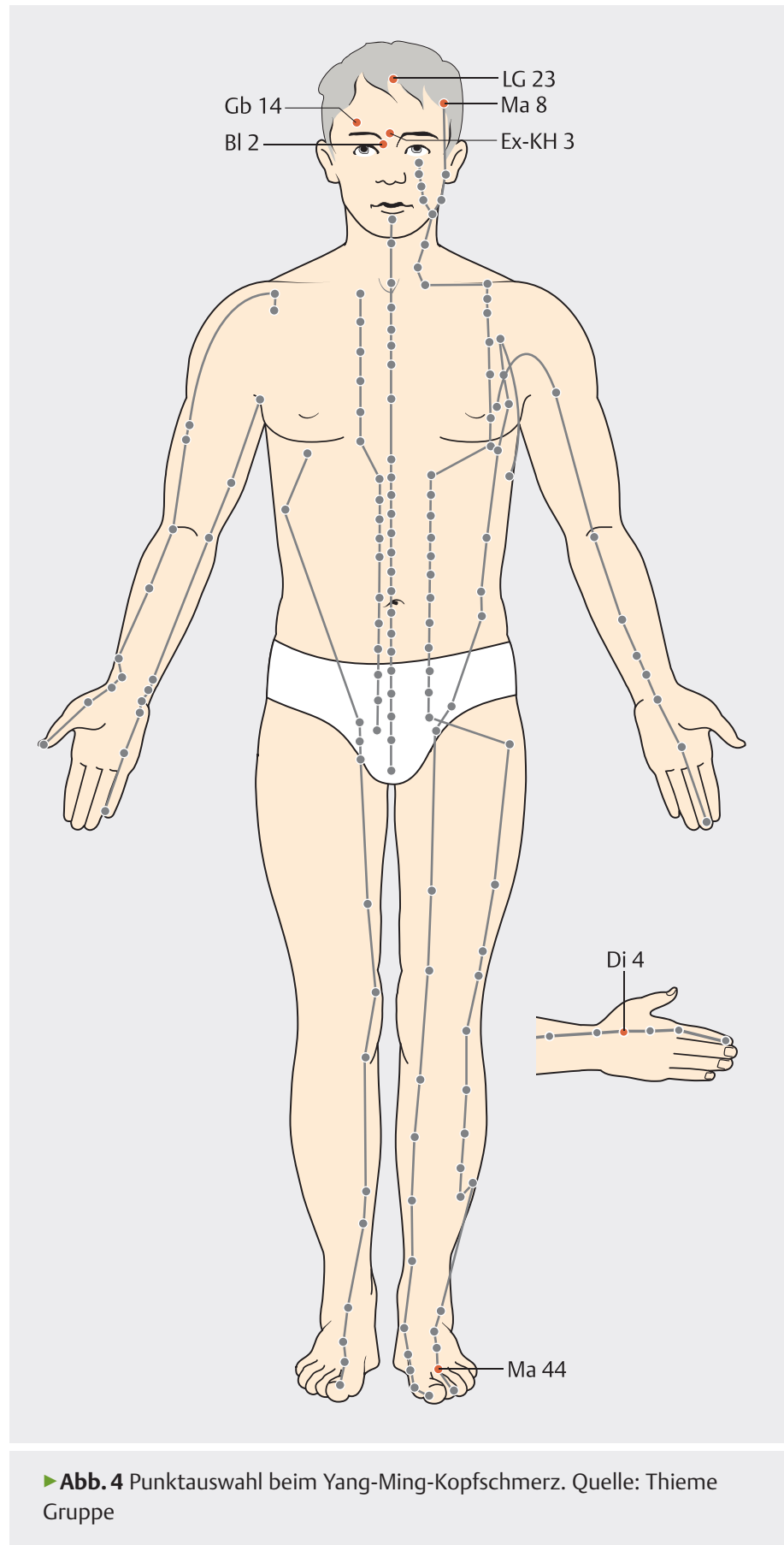

beim Leber-Blut-Mangel genadelt wird. Le 3 entspannt die Leber.

\section{Yang-Ming-Kopfschmerz}

Der Yang-Ming-Kopfschmerz korrespondiert mit der Magen- und Dickdarm-Leitbahn. Er manifestiert sich im Stirn- und gelegentlich Gesichtsbereich. Sowohl manche Spannungskopfschmerzen als auch mit Zahn- oder Kiefergelenksaffektionen assoziierte Kopfschmerzen werden hier zugeordnet, genauso wie Erkrankungen der Stirn- und Nebenhöhlen. Man beachte in diesen Fällen die Differenzialdiagnose zu Shao Yang (z. B. Gb 2, Gb 14) als auch zu Tai Yang (Bl 1 bis 3). 


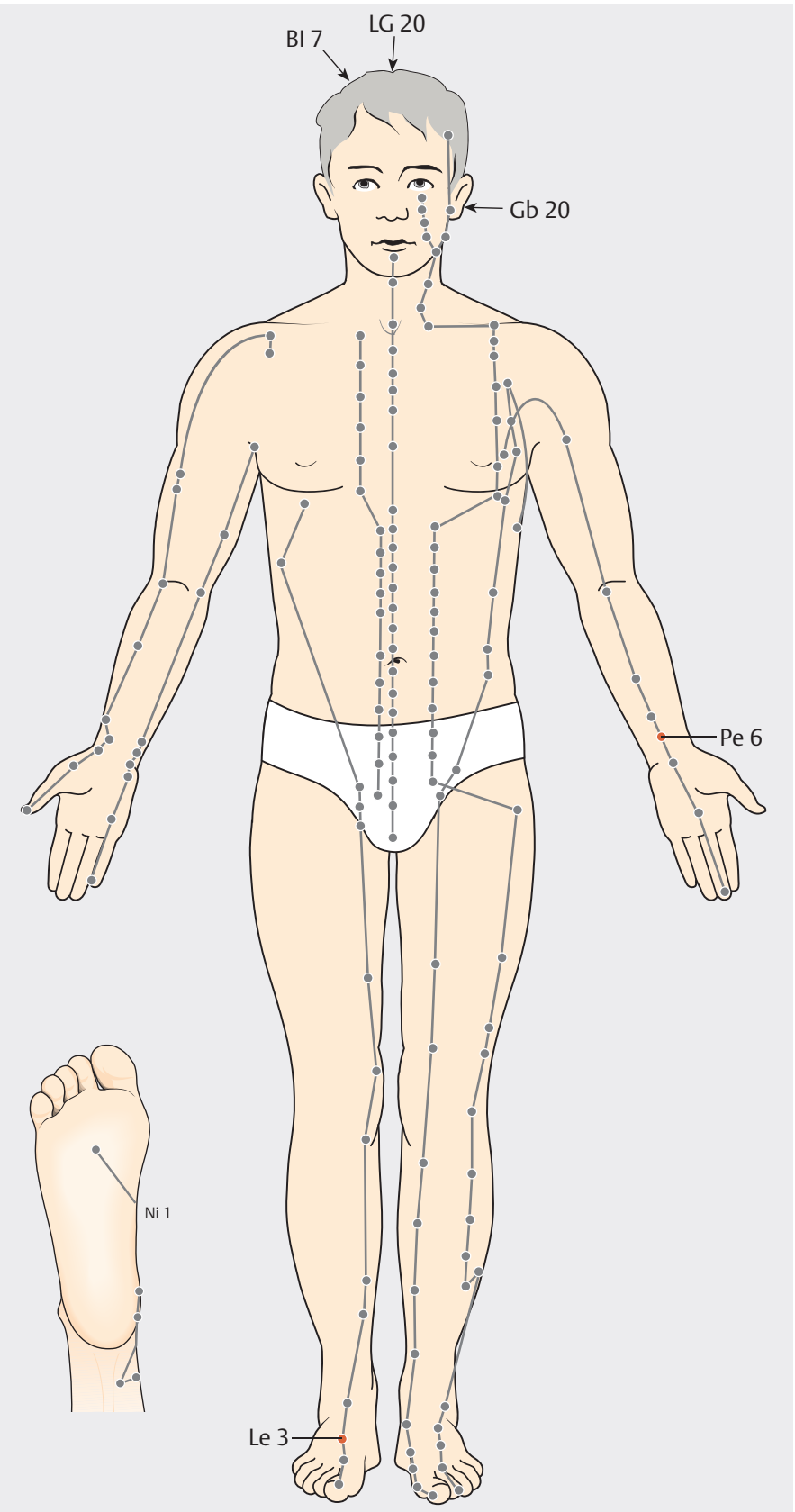

Abb. 5 Punktauswahl beim Jue-Yin-Kopfschmerz. Quelle: Thieme Gruppe eingeschränkte Nasenatmung. Ein angenehmer Nebeneffekt ist die den Shen-Geist beruhigende Wirkung, ohne müde zu machen. Ma 8 verbindet ähnlich wie Gb 14 die Magen-Leitbahn mit der Gallenblasen-Leitbahn und zusätzlich noch mit dem Yang Wei Mai. Daraus ergibt sich seine doppelte Indikation beim Yang-Ming- und Shao-Yang-Kopfschmerz. Ma 8 ist ein wichtiger Punkt, um Nässe und Schleim aus dem Kopf abzuleiten, die sich u. a. mit Benommenheit oder Schweregefühl manifestieren.

Di 4 ist der bekannteste Kopfschmerzpunkt. Er gehört zu den Kommandopunkten nach Gao Wu und ist der wichtigste Fernpunkt für alle Affektionen von Kopf und Gesicht. Zudem leitet Di 4 effektiv äußere pathogene Faktoren aus. Seine Lage auf der Dickdarm-Leitbahn macht ihn zum Hauptpunkt beim Yang-Ming-Kopfschmerz. Ma 44 ist der 2. Antike Ying-Punkt auf der Magen-Leitbahn. Diese Punktkategorie behandelt Erkrankungen der Yang-Leitbahnen, insbesondere Stagnation, und kühlt zusätzlich Hitze. Letzteres ist von Bedeutung, weil frontaler Kopfschmerz häufig aus Magen-Hitze resultiert.

\section{Jue-Yin-Kopfschmerz}

Der Jue-Yin-Kopfschmerz korrespondiert mehr mit der Leber-Leitbahn als mit der gekoppelten Perikard-Leitbahn. Ein innerer Ast der Leber-Leitbahn verläuft durch den Kopf bis zum Punkt LG 20. Daher manifestiert er sich als Scheitelkopfschmerz und hat meist innere Leber-Syndrome als Ursache. Differenzialdiagnostisch sollte man die Blasen-Leitbahn nicht vergessen, falls äußere pathogene Faktoren ursächlich sind oder der Kopfschmerz zum Nacken ausstrahlt.

Wichtige Akupunkturpunkte zur Behandlung des JueYin-Kopfschmerzes sind ( $\mathbf{A b b}$. 5):

- Lokal-/Nahpunkte: LG 20 (Bai Hui), Gb 20 (Feng Chi), BI 7 (Tong Tian), LG 23 (Shang Xing)

- Fernpunkte: Le 3 (Tai Chong), Pe 6 (Nei Guan), Ni 1 (Yong Quan)

LG 20 ist der zentrale Punkt auf dem Kopf. Er verbindet das Lenkergefäß mit der Leber-, Gallenblasen- und 3Erwärmer-Leitbahn. Folglich hat er eine breite Wirkung auf den Kopf und kann in Kombination mit Le 3 und Le 2 aufsteigendes Leber-Yang beruhigen. Wie der Extrapunkt Yin Tang beruhigt er auch den Geist-Shen, was bei ausgeprägten Leber- oder Gallenblasen-Pathologien hilfreich ist. BI 7 steht mit LG 20 in Verbindung, behandelt Kopfschmerz und vertreibt Wind. Alternativ können auch die Extrapunkte Si Shen Cong (Ex-KH 1) jeweils vor, hinter und neben LG 20 genadelt werden.

Le 3 hat über die Leber-Leitbahn neben seinen schon beschriebenen Wirkungen einen direkten Bezug zum Scheitel. Er ist hier sowohl leitbahnbezogener Fernpunkt als auch ein Punkt, der die ursächlichen Leber-Syndrome 
Tab. 3 Kopfschmerztypen und mögliche Ursachen aus der Perspektive der inneren Syndrome.

\begin{tabular}{|c|c|}
\hline Kopfschmerztyp & Zang-Fu-Syndrome \\
\hline Tai Yang (Bl, Dü) & $\begin{array}{l}\text { - Feuchte-Hitze in der Blase } \\
\text { - Leber-Qi-Stagnation } \\
\text { " } \text { aufsteigendes Leber-Yang } \\
\text { - Nieren-Yin-Mangel } \\
\text { " Nieren-Jing(Essenz)-Mangel }\end{array}$ \\
\hline Shao Yang (Gb, 3E) & $\begin{array}{l}\text { - Leber-Feuer } \\
\text { - Feuchte-Hitze in Leber/ } \\
\text { Gallenblase } \\
\text { - Leber-Qi-Stagnation } \\
\text { - Blut-Stase } \\
\text { " aufsteigendes Leber-Yang } \\
\text { " innerer Leber-Wind }\end{array}$ \\
\hline Yang Ming (Ma, Di) & $\begin{array}{l}\text { " Magen-Hitze } \\
\text { " Schleim-Feuchtigkeit } \\
\text { " Leber-Qi-Stagnation } \\
\text { " Leber-Feuer } \\
\text { " Disharmonie zwischen Gallen- } \\
\text { blase und Magen } \\
\text { " Milz-Qi-Mangel }\end{array}$ \\
\hline Jue Yin (Le, Pe) & $\begin{array}{l}\text { - Leber-Qi-Stagnation } \\
\text { " aufsteigendes Leber-Yang } \\
\text { - Leber-Blut-Mangel } \\
\text { - Herz-Blut-Mangel } \\
\text { - Qi- und Blut-Mangel }\end{array}$ \\
\hline
\end{tabular}

behandelt. Pe 6 liegt auf der innerhalb des Jue Yin gekoppelten Perikard-Leitbahn und unterstützt Le 3 . Le 3 mit Pe6 ist eine elegante, wirksame Punktkombination gegen Leber-Qi-Stagnation. Ni 1 spiegelt als tiefster Punkt des Körpers die höchste Region um LG 20. Er kann übermäßiges Yang aus dem Kopf nach unten leiten. Da seine Nadelung nicht immer schmerzfrei gelingt, wird er eher selten benutzt.

\section{Weitere Differenzierung von Kopfschmerzen}

In der TCM unterscheidet man zwischen der „Wurzel“ Ben einer Erkrankung und ihrem „Zweig“ Biao. Mit Biao meint man die symptomatische Manifestation und Ben benennt die Ursache. Wenn man z. B. einen Shao-YangKopfschmerz bei einer Leber-Qi-Stagnation diagnostiziert, ist die Schmerzmanifestation im Shao Yang der Zweig und das Leber-Syndrom die Wurzel. Mit den vorgestellten Therapieansätzen behandelt man primär Biao. Nur wenn die aufgrund der Schmerzlokalisation ausgewählten Punkte zufällig auch das ursächliche Syndrom ansprechen, wird Ben berücksichtigt.

Die Behandlung nach den 4 Kopfschmerztypen ist gut geeignet, um akute oder durch äußere pathogene Faktoren verursachte Kopfschmerzen zu behandeln. Bei chronisch rezidivierenden Kopfschmerzen und in schmerzfreien Intervallen ist eine weitere diagnostische Differenzierung und daraus folgende Modifikation dieser Punktkombinationen sinnvoll. > Tab. 3 gibt einen Überblick über die 4 Kopfschmerztypen und ihre möglichen Ursachen aus der Perspektive der inneren Syndrome.

Diese umfangreiche Auflistung zahlreicher und recht unterschiedlicher Syndrome kann anfangs etwas überfordern. Zusammenfassend lassen sich aber 5 grundlegende Pathomechanismen erkennen ( $\mathbf{A b b . 6 , ~}$ - Abb. 7, Abb. 8, - Abb. 9):

1. Eindringen äußerer pathogener Faktoren: Wind, Kälte, Hitze, Feuchtigkeit

2. Stagnation: Leber-Qi-Stagnation, Blut-Stase

3. Mangelversorgung mit Qi und/oder Blut-Xue: Z. B. Leber-Blut-Mangel, Herz-Blut-Mangel, Nieren-YinMangel, Milz-Qi-Mangel

4. übermäßiges Aufsteigen von Yang oder Feuer: z. B. Leber-Feuer, aufsteigendes Leber-Yang, Magen-Hitze

5. Akkumulation von Feuchtigkeit und Schleim

Eine detaillierte Darstellung der einzelnen Syndrome würde den gegebenen Rahmen sprengen. Deshalb wird im Folgenden anhand der Schmerzqualität und der Begleitsymptomatik eine vereinfachte Orientierung zur syndromorientierten Therapie gegeben. Allgemein gilt:

a. Die bereits beschriebene leitbahnbezogene Punktauswahl kann ganz oder teilweise beibehalten werden.

b. Ergänzt werden Punkte, die das zugrunde liegende Syndrom behandeln.

c. Bei Bedarf können zusätzlich Ahshi- oder auch Triggerpunkte genadelt werden, um die lokale Stagnation zu lösen.

d. Im akuten Fall haben die Punkte unter a) Priorität, in chronischen Fällen oder im schmerzfreien Intervall sind die Punkte unter b) wichtiger.

\section{Eindringen von äußeren pathogenen Faktoren}

Dabei handelt es sich um Fülle-Zustände, die meist akut und plötzlich auftreten. Sie betreffen die 3 Yang-Achsen Tai Yang, Shao Yang, Yang Ming oder auch den ganzen Kopf. Es können weitere äußere Symptome auftreten, wie z. B. Frösteln, Fieber, Wind- oder Kälteempfindlichkeit, Muskelschmerzen und Erkältungssymptome. Der Puls kann oberflächlich sein und der Zungenbelag etwas verdickt. Bei Wind-Hitze können noch ein Hitzegefühl oder Rötung von Gesicht und Augen dazu kommen. Bei äußerer Feuchtigkeit fühlt sich der Kopf schwer und wie in Watte gepackt an. Es kann zu vermehrter nasaler Schleimabsonderung kommen. Verschlimmerung tritt meist bei feuchtem Wetter ein.

Meist sind die Punktkombinationen nach den 4 Kopfschmerztypen hier schon sehr effektiv, da viele der genannten Punkte auch äußere pathogene Faktoren ausleiten. Alle Punkte werden mit sedierender Nadeltechnik gestochen. Di 4 (Hegu) kann in allen Fällen, unabhängig 


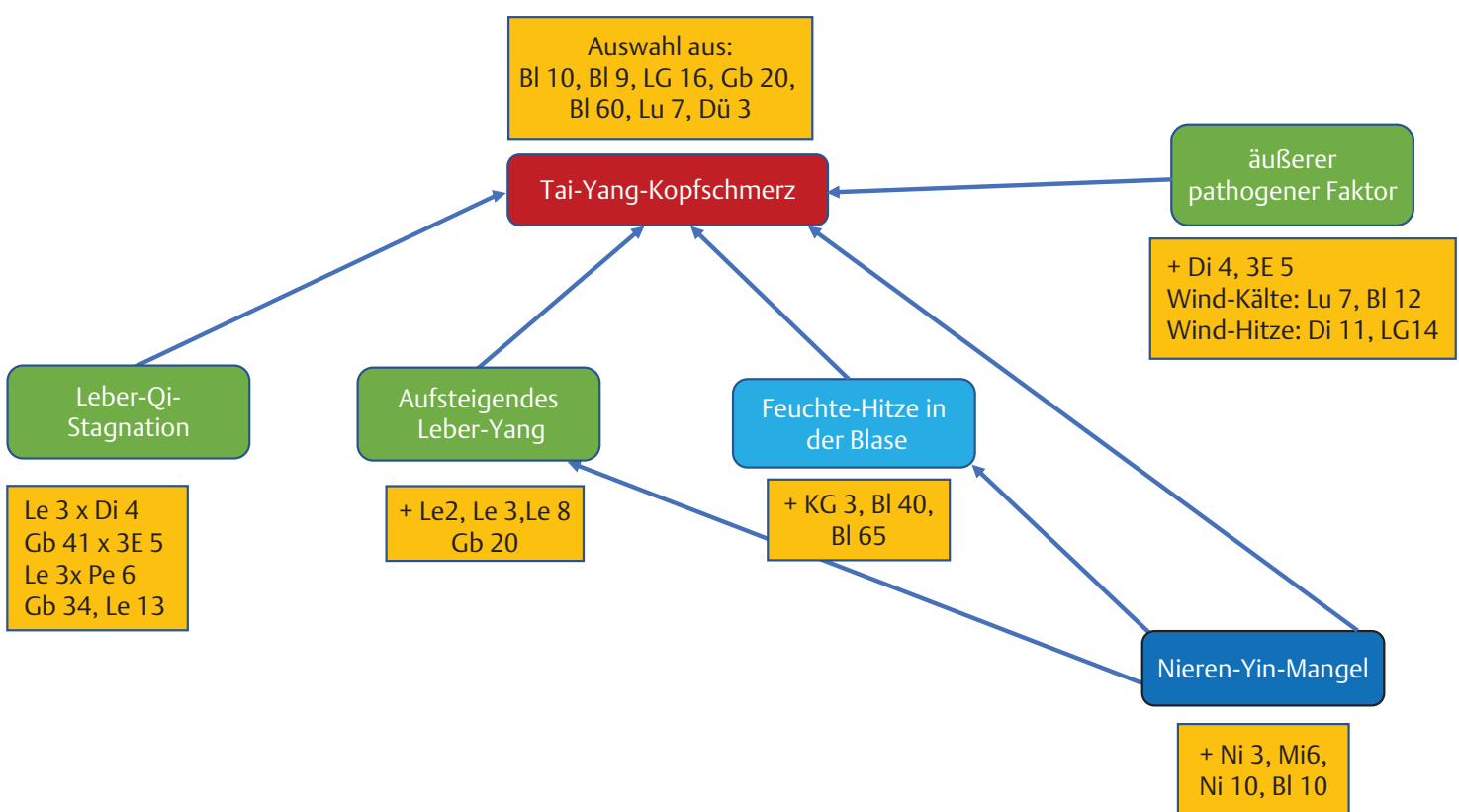

- Abb. 6 Therapie beim Tai-Yang-Kopfschmerz. Quelle: Dr. med. Stefan Kirchhoff

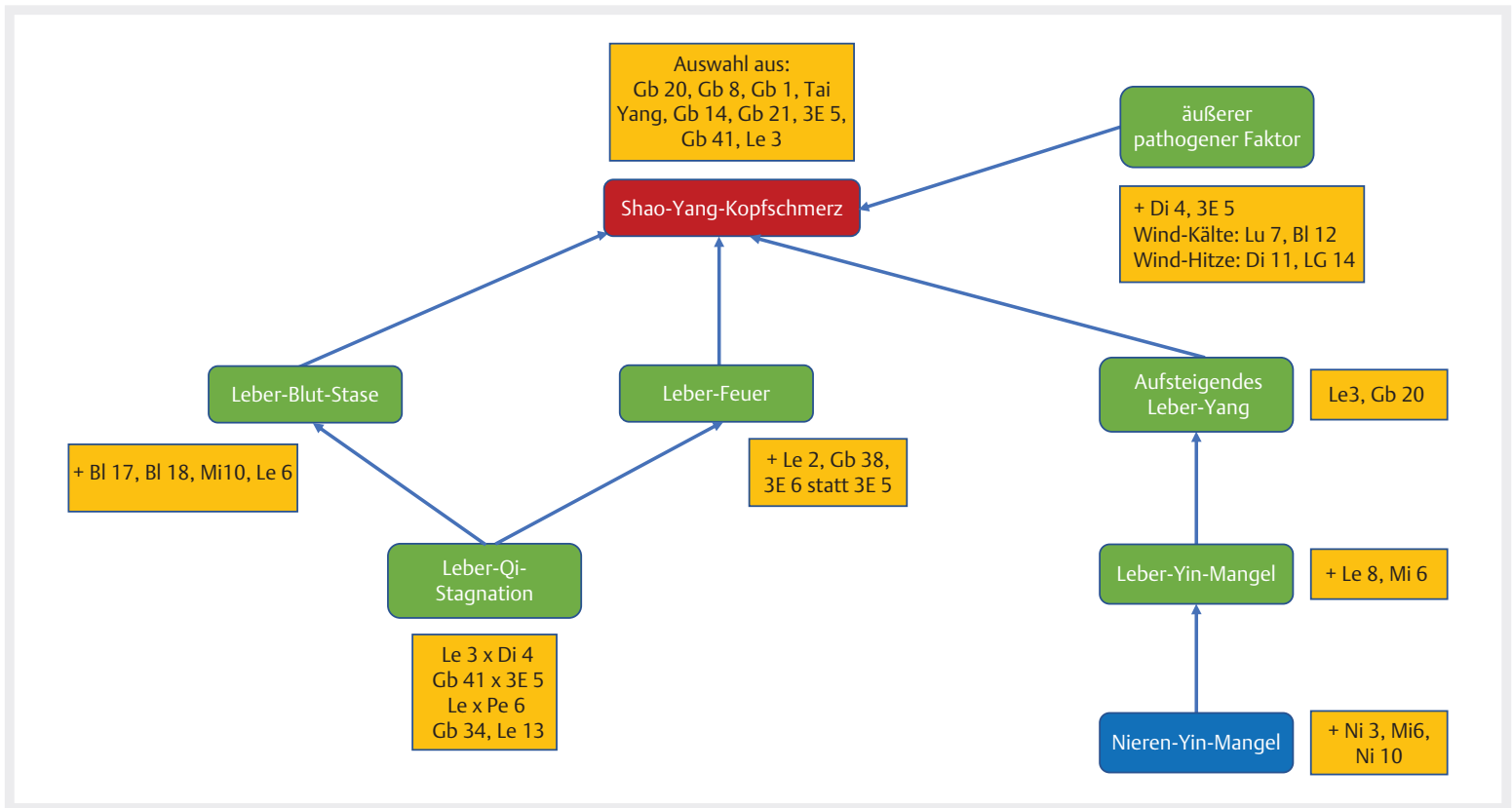

Abb. 7 Therapie beim Shao-Yang-Kopfschmerz. Quelle: Dr. med. Stefan Kirchhoff

von der Kopfschmerzlokalisation, hinzugefügt werden, da er einer der Hauptpunkte zur Ausleitung von pathogenen Faktoren ist.

Bei Wind-Kälte-Kopfschmerzen können ergänzend Lu 7 (Lie Que) und/oder Bl 12 (Feng Men) genadelt werden.
Wind-Kälte dringt über die Poren der Haut, die von der Lunge kontrolliert werden, ein und blockiert oft die oberflächlichen Luo-Gefäße. Lu 7 ist der Luo-Punkt der Lungen-Leitbahn und vertreibt Wind-Kälte. Der chinesische Name von Bl 12 bedeutet „Tor des Windes“ und beschreibt die Fähigkeit dieses Punkts, pathogenen 


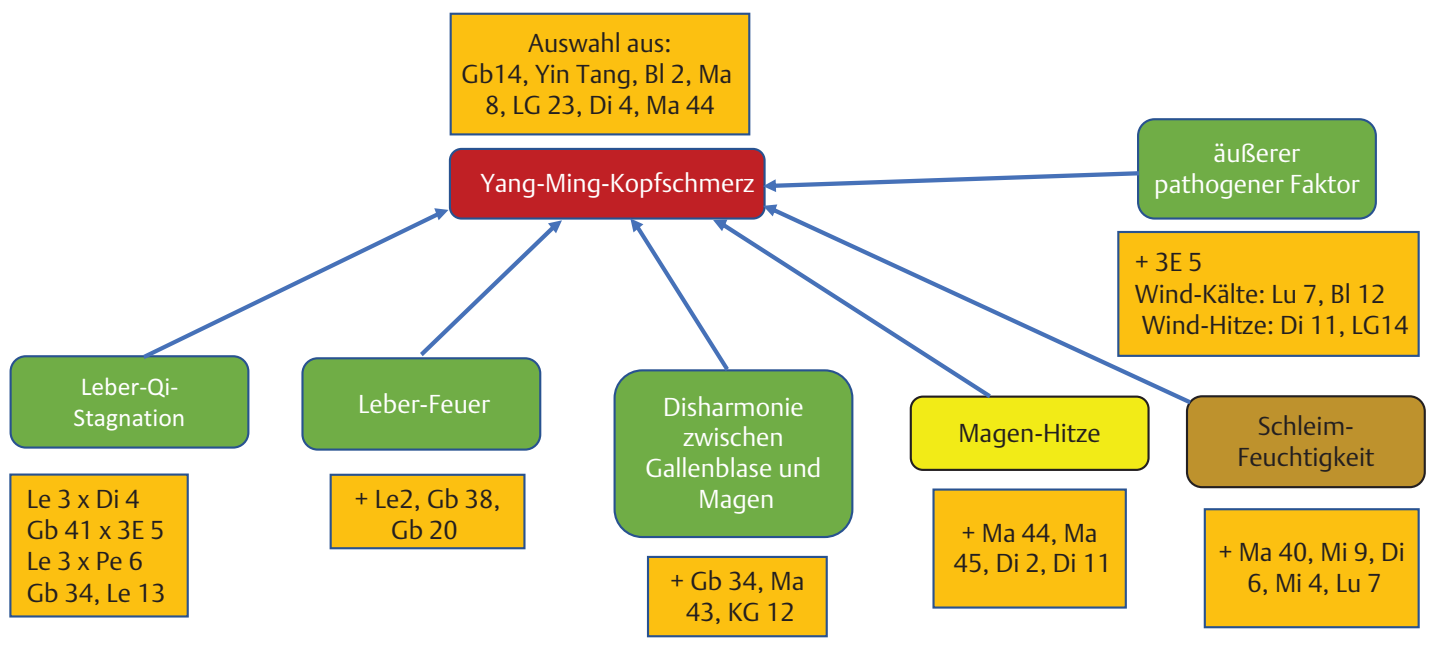

- Abb. 8 Therapie beim Yang-Ming-Kopfschmerz. Quelle: Dr. med. Stefan Kirchhoff

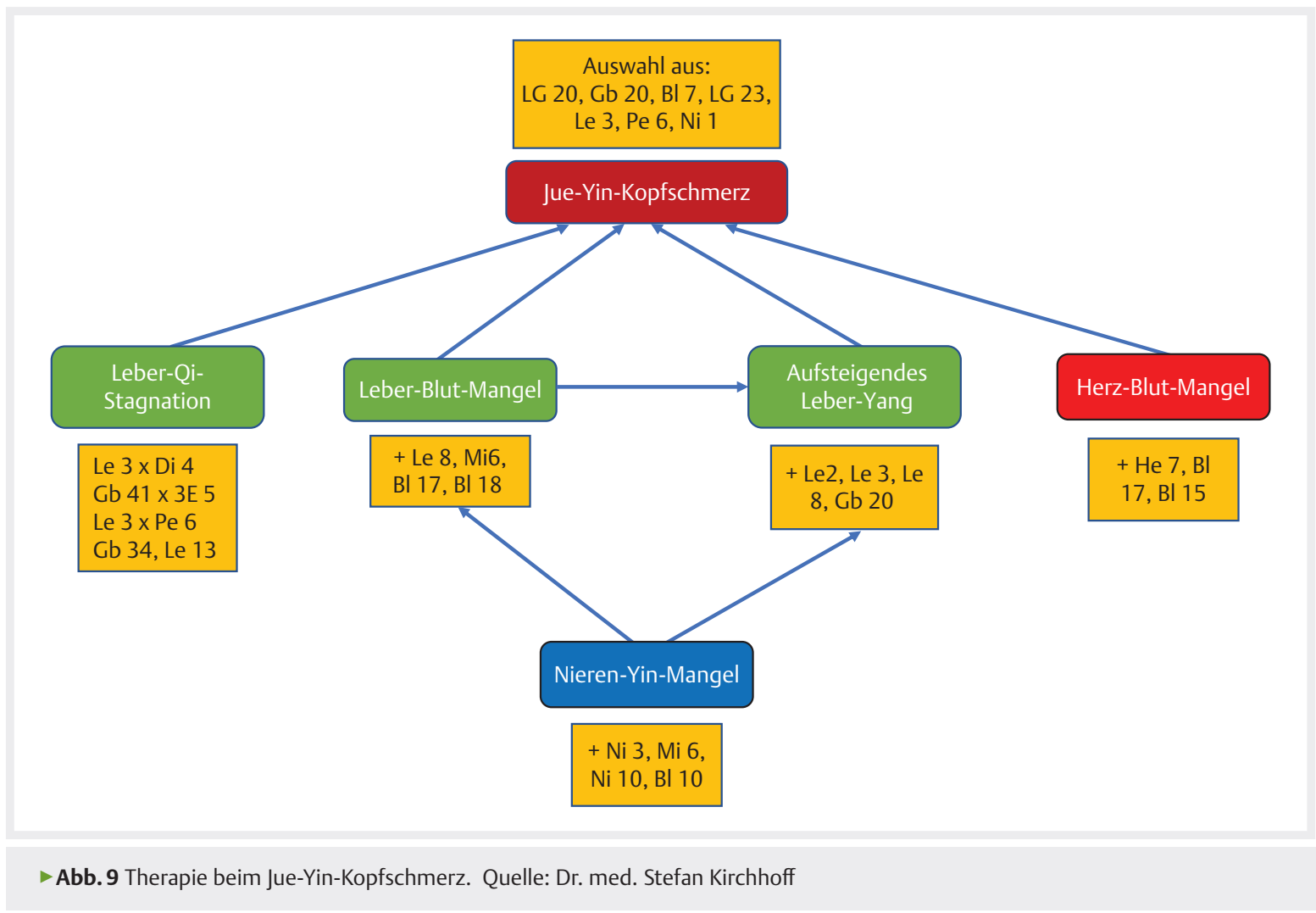

äußeren Wind auszuleiten. Zusätzlich kann in der Region von Bl 12 und Bl 13 Gua Sha appliziert werden.

Bei Wind-Hitze-Kopfschmerzen können ergänzend Di 11 (Qu Chi) und LG 14 (Da Zhui) genadelt werden. Di 11 ist zwar kein Punkt gegen Kopfschmerzen, aber gut geeignet, um Hitze zu kühlen. LG 14 ist ein Treffpunkt aller
Yang-Leitbahnen, die von dort zum Kopf ziehen, er kühlt Hitze, vertreibt Wind und lindert Kopfschmerz. Di 11 mit LG 14 ist eine klassische Kombination gegen Wind-Hitze.

Bei Wind-Feuchtigkeit-Kopfschmerzen können ergänzend Lu 7 (Lie Que), $3 E 4$ (Yang Chi) und Mi 9 (Yin Ling Quan) genadelt werden. Lu 7 als Luo-Punkt leitet 
Feuchtigkeit aus dem oberen Erwärmer aus. 3E 4 ist der Yuan-Quellpunkt und reguliert die Funktion des 3-Erwärmers. Dieser kontrolliert die Flüssigkeitswege und zusammen mit der Milz einen Teil des Flüssigkeitsmetabolismus. Mi 9 leitet nicht äußere, sondern innere Feuchtigkeit aus. Länger bestehende äußere Feuchtigkeit kann aber durchaus die Milz-Funktion belasten, insbesondere, wenn der Patient schon eine Feuchtigkeitskonstitution besitzt.

\section{Stagnation von Qi und/oder Blut-Xue}

Der vermutlich häufigste Pathomechanismus bei Kopfschmerzen im klinischen Alltag ist die Leber-Qi-Stagnation. Sie kann für jeden der 4 Kopfschmerztypen verantwortlich sein, hat jedoch eine Präferenz für den temporalen und frontalen Kopfschmerz. Er kann einoder beidseitig auftreten und auch den ganzen Kopf betreffen. Mögliche schulmedizinische Diagnosen umfassen Migräne, Spannungskopfschmerz, Kopfschmerzen bei Hypertonie oder den prämenstruellen Kopfschmerz. Der Kopfschmerz ist oft mit emotionalen Belastungen oder Stress assoziiert, kann aber auch in Entspannungsphasen wie an Wochenenden gehäuft auftreten. Weitere Symptome bei Leber-Qi-Stagnation sind u. a. Reizbarkeit, Launenhaftigkeit oder depressive Verstimmtheit, nächtliches Zähneknirschen, Dysmenorrhö, abdominales Völleoder Spannungsgefühl. Der Puls ist drahtig und die Zunge kann aufgerollte Zungenränder zeigen. Für die Vielzahl anderer möglicher Symptome sei auf die gängige Literatur verwiesen.

Viele der gegen den Shao-Yang- und Jue-Yin-Kopfschmerz empfohlenen Punkte sind unabhängig von der Lokalisation auch bei der Leber-Qi-Stagnation indiziert: Gb 20, LG 20, Gb 14, Le 3, Pe 6, 3E5. Le 3 ist der wichtigste Punkt bei Leber-Qi-Stagnation. Er wird oft beidseits zusammen mit Di 4 genadelt. Diese klassische Kombination ist als die „4 Tore“ bekannt. Sie beseitigt Leber-Qi-Stagnation, entspannt emotional und lindert Schmerz. Obwohl sehr effektiv, ist die Wirkung der „4 Tore“ eher oberflächlich und nicht allzu nachhaltig. Für einen dauerhaften Erfolg müssen noch andere Strategien hinzugenommen werden. Alternativ oder ergänzend zu den „4 Toren“ kann die Öffnung des Dai Mai mit Gb 41 und kontralateral 3E 5 oder die Kombination Le 3 und Pe 6 sinnvoll sein. Gb34 (Yang Ling Quan) bewegt das Leber-Qi. Er ist besonders dann indiziert, wenn die Leber-Stagnation mit ausgeprägten muskulären Verspannungen einhergeht. Le 13 (Zhang Men) ist der Mu-Punkt der Leber und bewegt Leber-Qi.

Bei einer chronischen Leber-Qi-Stagnation mit rezidivierend auftretenden Kopfschmerzen ist Akupunktur für eine dauerhafte Besserung meist nicht ausreichend. Die Leber-Qi-Stagnation ist oft eine Folge der persönlichen Lebensumstände und Gewohnheitsmuster. Sie hat viel mit der Wahrnehmung und Interpretation der Welt und der Art der Stress- und Konfliktbewältigung zu tun. In diesen Fällen ist die aktive Mitarbeit des Patienten, in manchen Fällen unter begleitender Psychotherapie, erforderlich.

Innere Zang-Fu-Syndrome treten selten einzeln auf. Gerade bei chronischen Beschwerden findet man häufig mehrere Syndrome, die sich im zeitlichen Verlauf entwickelt haben und nun gleichzeitig mit ihren jeweiligen Symptomen auftreten. Insbesondere gilt dies für die Leber-Syndrome. So kann Leber-Qi-Stagnation z. B. zu Leber-Feuer oder Milz-Qi-Mangel führen oder aber auch durch einen Leber-Blut-Mangel begünstigt werden. Diese Zusammenhänge sind für die korrekte Punktauswahl wichtig. Eine Punktkombination sollte das Syndrommuster eines Patienten so gut wie möglich abbilden.

\section{Blut-Stase}

Eine chronische Leber-Qi-Stagnation kann sich zu einer Leber-Blut-Stase weiterentwickeln. Akut kann eine BlutStase die Folge eines lokalen Traumas sein. Differenzialdiagnostisch sind intrakraniale Blutungen oder Tumoren auszuschließen. Der Schmerz ist intensiv, stechend, bohrend mit fester, durch den Patienten klar zu benennender, punktförmiger Lokalisation. Allgemeine Blut-StaseZeichen, z.B. Dysmenorrhö mit großen Blutkoageln, können, müssen aber nicht vorhanden sein. Auch bislang therapieresistente, chronische Kopfschmerzen können mit einer Blut-Stase zusammenhängen.

Neben Punkten für die 4 Kopfschmerztypen, und im Fall von begleitender Leber-Qi-Stagnation den dafür indizierten Punkten, kann man zusätzlich blutbewegende Punkte nadeln. Ahshi-Punkte lösen die Blut-Stase lokal. Bl 17 (Ge Shu) ist der Hui-Meister-Punkt des Bluts und bewegt das Blut systemisch. Mi 10 (Xue Hai) trägt den Namen „Meer des Bluts“ und bewegt und harmonisiert das Blut. Xi-Akkumulationspunkte auf den jeweils betroffenen Leitbahnen haben eine Qi- und blutbewegende Wirkung und sind besonders bei akuten Schmerzen indiziert.

\section{Mangelversorgung des Kopfs}

Hier finden wir vornehmlich Qi- und Blut-Mangel-Syndrome. Auch Yang- oder Yin-Mangel sind möglich, wobei Letzterer meist zu aufsteigendem Yang führt. Der Kopfschmerz ist meist chronisch, eher im Hintergrund mit einer dumpfen Schmerzqualität und kann auch den ganzen Kopf betreffen. Im Vergleich zum Fülle-Kopfschmerz ist er besser zu tolerieren. Qi-Mangel korreliert meist mit Milz und Lunge und Blut-Mangel mit Leber oder Herz. Qi- und Blut-Mangel können durchaus gemeinsam auftreten; die Unterschiede zwischen ihnen zeigt $\triangleright$ Tab. 4 .

Primär ist der Mangel aufzufüllen. Die symptomatische Beseitigung von akutem Kopfschmerz durch z. B. Lösen 
> Tab. 4 Differenzierung von Qi- und Blut-Mangel-Kopfschmerz.

\begin{tabular}{|c|c|c|}
\hline & Qi-Mangel-Kopfschmerz & Blut-Mangel-Kopfschmerz \\
\hline bevorzugte Lokalisation & $\begin{array}{l}\text { " ganzer Kopf } \\
\text { " } \text { frontal } \\
\text { " schwierig zu lokalisieren }\end{array}$ & $\begin{array}{l}\text { " } \text { Vertex } \\
\text { " hinter den Augen } \\
\text { " ganzer Kopf }\end{array}$ \\
\hline Verschlechterung & $\begin{array}{l}\text { " nach Anstrengung } \\
\text { " gegen Abend }\end{array}$ & $\begin{array}{l}\text { " } \text { nach Anstrengung } \\
\text { " gegen Abend } \\
\text { " nach Menstruation (Blutverlust) } \\
\text { " durch Entbindung } \\
\text { der Augen Bildschirmarbeit oder andere Belastungen }\end{array}$ \\
\hline Zunge & blass, geschwollen, evtl. Zahnabdrücke & blass, flach, evtl. blasse Zungenränder \\
\hline sonstige Symptome & $\begin{array}{l}\text { " Erschöpfung, Müdigkeit } \\
\text { " schwacher Muskeltonus } \\
\text { " blasses Gesicht } \\
\text { " Bedürfnis, den Kopf bei Kälte oder } \\
\text { Wind zu bedecken } \\
\text { - } \text { weitere Qi-Mangel-Symptome }\end{array}$ & $\begin{array}{l}\text { " nächtliches Erwachen mit Kopfschmerzen } \\
\text { " akute Exazerbationen sind möglich } \\
\text { " blasses, glanzloses Gesicht } \\
\text { " Schlafstörungen } \\
\text { - } \text { weitere Blut-Mangel-Symptome }\end{array}$ \\
\hline
\end{tabular}

- Tab. 5 Differenzierung der Hitze-Syndrome bei Kopfschmerzen.

\begin{tabular}{|c|c|c|c|}
\hline & Leber-Feuer & Magen-Hitze & aufsteigendes Leber-Yang \\
\hline Lokalisation & $\begin{array}{l}\text { - } \text { temporal (Shao Yang) } \\
\text { " } \text { frontal (Yang Ming) } \\
\text { " hinter den Augen }\end{array}$ & frontal (Yang Ming) & $\begin{array}{l}\text { " } \text { temporal (Shao Yang) } \\
\text { " Vertex (Jue Yin) } \\
\text { " hinter den Augen }\end{array}$ \\
\hline Verschlechterung & $\begin{array}{l}\text { - Stress } \\
\text { " Ärger } \\
\text { - Alkohol } \\
\text { - scharfe Speisen }\end{array}$ & $\begin{array}{l}\text { " scharfe Speisen } \\
\text { - Alkohol }\end{array}$ & $\begin{array}{l}\text { " chronischer Stress } \\
\text { - Schlafmangel }\end{array}$ \\
\hline Besserung & $\begin{array}{l}\text { - Erbrechen } \\
\text { - kalte Umschläge }\end{array}$ & kalte Umschläge auf der Stirn & kalte Umschläge \\
\hline Zunge & rot, gerötete Ränder, gelber Belag & rot, gelber Belag & $\begin{array}{l}\text { rot, wenig oder fehlender Belag, evtl. } \\
\text { rissig }\end{array}$ \\
\hline sonstige Symptome & $\begin{array}{l}\text { - Gesichtsrötung } \\
\text { " gerötete Augen } \\
\text { " bitterer Mundgeschmack } \\
\text { " Übelkeit und Erbrechen mit } \\
\text { Linderung } \\
\text { " Reizbarkeit, cholerisches } \\
\text { Verhalten }\end{array}$ & $\begin{array}{l}\text { " Magenschmerz } \\
\text { " Heißhunger } \\
\text { " permanent hungrig } \\
\text { " Reflux } \\
\text { " Zahnfleischentzündung und } \\
\text {-bluten } \\
\text { " Aphten } \\
\text { " Mundgeruch } \\
\text { " Durst auf kalte Getränke } \\
\text { " Verstopfung }\end{array}$ & $\begin{array}{l}\text { - Unruhe, Reizbarkeit } \\
\text { - Benommenheit } \\
\text { " Sehstörungen, Lichtempfindlichkeit } \\
\text { - Übelkeit und Erbrechen ohne } \\
\text { Linderung } \\
\text { - Erschöpfung }\end{array}$ \\
\hline
\end{tabular}

von Stagnation oder Vertreiben von pathogenen Faktoren ist sekundär. Deshalb sind die tonisierenden Fernpunkte wichtiger als die leitbahnbezogenen Konzepte. Die entsprechenden Punkte werden mit tonisierender Nadeltechnik (Bu Fa) gestochen. Dennoch können bei eindeutiger Lokalisation auch Punkte aus den Kombinationen der 4 Kopfschmerztypen dazugenommen werden.

\section{Qi-Mangel-Kopfschmerz}

Bei Qi-Mangel wird das Qi von Milz und Lunge gestärkt. Ma 36 (Zu San Li) ist der 5. AntikeHe-Punkt und der Erde-Punkt. Er wirkt direkt auf Magen und Milz. Er tonisiert das Qi und Blut-Xue, indem er die Umwandlung von Nahrung und Flüssigkeiten in Qi fördert. Als Punkt auf der Yang-Ming-Achse ist er besonders geeignet, frontalen Yang-Ming-Kopfschmerz zu behandeln, solange 
dieser nicht durch volle Magen-Hitze verursacht ist. Di 10 (Shou San Li) ist das Pendant zu Ma 36 am Arm. Er tonisiert das Qi und leitet es zu den oberen Körperbereichen. Somit ist Di 10 ein guter Punkt, um Ma 36 in einer Kombination zu verstärken. Lu 9 (Tai Yuan) ist der YuanQuellpunkt, der Erde-Punkt, der 3. Antike-Shu-Punkt. Als Yuan-Pukt auf einer Yin-Leitbahn hat er einen stärkenden Einfluss direkt auf das Zang Lunge. Als Erde-Punkt ist er auch der Tonisierungspunkt der Lunge. Erde ist die Mutter des Metalls. Dieser Punkt stärkt somit hier das Lungen-Qi. KG 6 (Qi Hai) kann bei allen Mangel-Zuständen des Qi tonisierend genadelt werden. Sein Name bedeutet in der Übersetzung „Meer des Qi“. BI 13 (Fei Shu) und BI 20 (Pi Shu) sind die Rücken-Shu-Punkte von Lunge und Milz und tonisieren die ihnen zugeordnete Organe. LG 20 (Bai Hui) kann in diesem Zusammenhang tonisierend oder mit Stichrichtung nach frontal genadelt werden, um Qi und Yang nach oben zum Kopf zu ziehen.

Die genannten Punkte können auch mit Moxa behandelt werden, insbesondere, wenn zusätzlich ein Yang-Mangel mit Kälte-Symptomen vorliegt. In diesem Fall würden auch Nieren-Punkte wie Ni3 (Tai Xi) und Bl 23 (Shen Shu) ergänzt.

\section{Blut-Mangel-Kopfschmerz}

Ma 36 (Zu San Li) stärkt Qi und Blut über die Umwandlung der Nahrung. Le 8 (Qu Quan) ist der Wasser-Punkt und somit der Tonisierungspunkt der Leber. Er nährt das Leber-Yin und -Blut. Le 3 (Tai Chong) als 3. Antiker ShuPunkt, Erde-Punkt und Yuan-Punkt reguliert die Leber und nährt das Leber-Blut. Die Kombination von Le 3 und dem Rücken-Shu-Punkt Bl 18 (Gan Shu) hat einen direkt tonisierenden Einfluss auf die Leber. Ergänzt man nun noch Bl 17 (Ge Shu) als Hui-Meisterpunkt des Bluts zu diesen beiden Punkten, definiert man die Wirkung dieser Kombination als Blut nährend. Gb 39 (Xuan Zhong) ist der Hui-Meisterpunkt des Marks und berücksichtigt die Produktionskette des Bluts. Diese beginnt mit der Essenz-jing, zu dem das Mark gerechnet wird.

Die Produktion von Blut-Xue benötigt Qi und ist von den Nieren abhängig. Deshalb werden zusätzlich das Qi tonisierende und die Nieren stärkende Punkte genadelt: KG 4 (Guan Yuan), Ni 3 (Tai Xi), BI 20 (Pi Shu) und BI 23 (Shen Shu). Falls sich der Kopfschmerz auf dem Vertex manifestiert, ergänzt man Punkte wie Pe 6 (Nei Guan) oder LG 20 (Bai Hui).

\section{Übermäßiges Aufsteigen von Yang oder Feuer}

Es gibt 2 Mechanismen, die zu Kopfschmerzen dieses Typs führen. Der eine ist, dass eine Yang-Fülle in einem Organ wie Leber oder Magen seiner Natur folgend als Hitze nach oben drängt. Ursache in Bezug auf die Leber ist oft eine Leber-Qi-Stagnation mit unterdrückten Emotionen und/oder eine entsprechende Yang-Konstitution in der Wandlungsphase Holz. Alkohol und scharfe
Speisen können die Entwicklung von Leber-Feuer begünstigen und für Magen-Hitze ursächlich sein. Der andere ist, dass das Yin von Leber und/oder Niere zu schwach ist, um das physiologische Yang an seinem Platz zu halten, sodass es nach oben aufsteigen kann. Die Kopfschmerzen treten meist anfallsartig auf und besitzen eine scharfe, pulsierende, berstende oder klopfende Qualität. Die Unterschiede der einzelnen Syndrome zeigt $>$ Tab. 5 .

Die Therapieprinzipien umfassen neben der Schmerzstillung und Stagnationslösung im akuten Anfall auch die Behandlung der zugrunde liegenden Muster durch Hitze Klären, Yang Absenken und, wenn nötig, Yin Stärken. Im akuten Anfall können die 4 Kopfschmerztypen als Orientierung für die Punktauswahl dienen. Sie werden dann durch die folgenden Punkte modifiziert. Im schmerzfreien Intervall ist es dann umgekehrt, die syndromorientierten Punkte bestimmen die Therapie und werden durch lokale und distale Punkte der Achsen modifiziert. Je nach Yang-Fülle oder Yin-Mangel wird sedierend oder tonisierend genadelt. Moxa kommt nicht zum Einsatz.

Bei Leber-Feuer ergänzt man Le2 (Xing Jian), Gb 38 (Yang Fu) und ersetzt 3E 5 durch 3 E 6 (Zhi Gou). Cave: Lokale Punkte oberhalb von Gb 20 können im akuten Anfall zu einer Verschlimmerung führen. Le 2 ist der 2. Antike Ying-Punkt und kühlt Hitze in der Leber. Als Feuer-Punkt auf einer Holz-Leitbahn ist er auch der Sedierungspunkt und reduziert Leber-Feuer. Gb 38 ist auch ein Feuer-Punkt und somit der Sedierungspunkt der Gallenblase. Die Fülle der Leber fließt häufig in die Gallenblasen-Leitbahn über, weshalb es zum Shao-YangKopfschmerz kommt. $3 E 6$ als Feuer-Punkt wird sedierend genadelt und klärt Hitze im Shao Yang.

Bei aufsteigendem Leber-Yang hingegen ist zu beachten, dass ein Leber-Yin-Mangel zugrunde liegt. Mehr noch, hinter dem Leber-Yin-Mangel ist oft auch ein Nieren-Yin-Mangel zu finden. Man hat also häufig ein komplexes Bild, bei dem ein Nieren-Yin-Mangel zu LeberYin-Mangel mit aufsteigendem Leber-Yang und Fülle in der Gallenblasen-Leitbahn führen kann. Dementsprechend nadelt man z. B. Ni 3 (Tai Xi), Mi 6 (San Yin Jiao) und Le 8 (Qu Quan). Im Anfall können auch noch Le 2 und Gb38 gestochen werden. Ni3 ist der Erde-Punkt der Niere und stärkt u. a. das Nieren-Yin. Mi 6 als Treffpunkt der 3 Yin-Leitbahnen am Bein verbindet Leber und Niere und nährt das Yin. Le 8 ist der Wasser- und somit Tonisierungspunkt auf der Leber-Leitbahn. Er tonisiert das Leber-Yin.

Bei Magen-Hitze werden die Punkte des Yang-MingKopfschmerzes genadelt, von denen Ma 44 (Nei Ting) als 2. Antiker Ying- und Wasser-Punkt der wichtigste ist. Zusätzlich ist die Nadelung von KG 12 (Zhong Wan), Di 11 (Qu Chi), Di 2 (San Jian) und Pe 6 (Nei Guan) indiziert. 
KG 12 als Mu-Alarmpunkt des Magens reguliert den Magen und kann Hitze im Magen kühlen. Di 11 und Di 2 sind beides Punkte auf der Yang-Ming-Achse, die über die Klärung von Hitze im Dickdarm auch den Magen entlasten. Pe 6 kühlt zwar nicht direkt Hitze, hat aber über die Mittag-Mitternacht-Regel und als Kommandopunkt für Thorax und oberes Abdomen einen starken harmonisierenden Einfluss auf den Magen.

\section{Akkumulation von Feuchtigkeit und Schleim}

Hier handelt es sich um ein chronisches inneres Syndrom, das nicht mit dem Kopfschmerz durch WindFeuchtigkeit verwechselt werden sollte. Häufig gibt es eine wechselseitige Beziehung zum Milz-Qi-Mangel, der die Akkumulation von Feuchtigkeit und die Entstehung von Schleim begünstigt. Andererseits führt eine chronische Belastung durch Feuchtigkeit und Schleim auch zu einer Schwächung der Milz-Funktion.

Der Kopfschmerz ist oft dumpf oder drückend. Typisch ist das Schweregefühl oder das Gefühl, als wenn der Kopf in Watte eingepackt wäre. Betroffen ist meist der ganze Kopf oder der frontale Bereich, was sich über die Verbindung Milz-Magen erklärt. Weitere mögliche Symptome sind u. a.:

- Benommenheit, weil Schleim die Sinnesorgane behindert

- Lethargie mit schwerem Körpergefühl

- Appetitmangel oder Übelkeit, wenn durch Feuchtigkeit und Schleim in der Mitte das Absinken des Magen-Qi gehemmt wird

- Müdigkeit durch den begleitenden Qi-Mangel

- geschwollene Zunge mit dickem fettigem Belag

Schleim muss transformiert werden und Feuchtigkeit wird ausgeleitet. Bei begleitendem Milz-Qi-Mangel wird die Transformationsfunktion der Milz gestärkt. Folgende Punkte kommen in Frage: Ma 40 (Feng Long), Mi 9 (Yin Ling Quan), Mi 4 (Gong Sun), Lu 7 (Lie Que), Ma 8 (Tou Wei), KG 12 (Zhong Wan), Di 4 (He Gu). Bei frontaler Manifestation können Punkte aus dem Konzept für YangMing-Kopfschmerzen ergänzt werden.

\section{Interessenkonflikt}

Der Autor gibt an, dass kein Interessenkonflikt besteht.

\section{Autorinnen/Autoren}

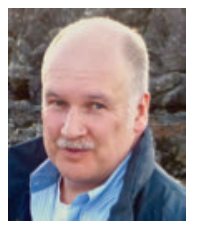

\section{Stefan Kirchhoff}

ist Facharzt für Allgemeinmedizin und in eigener privatärztlicher Praxis für TCM niedergelassen. Beschäftigung mit TCM seit 40 Jahren. Zusammen mit Dr. Elmar Peuker wissenschaftliche Leitung der Weiterbildung Akupunktur der Akademie für ärztliche Fortbildung der ÄKWL. Lehrauftrag für Akupunktur an der Ruhr-Universität Bochum.

\section{Korrespondenzadresse}

Dr. med. Stefan Kirchhoff

Oststr. 38

45549 Sprockhövel

E-Mail: kirchhoff.tcm@t-online.de

\section{Literatur}

[1] Deadman P, Al-Khafaji M, Baker K. Handbuch der Akupunktur. 3. Aufl. Kötzting Wald: Systemische Medizin; 2012

[2] Maclean W, Lyttleton J. Clinical Handbook of Internal Medicine. Campletown: University of Western Sydney MacArtur; 1998

[3] Sun P. Treatment of Pain with Chinese Herbs and Acupuncture. Edinburgh: Churchill Livingstone; 2010

[4] Montakab H. Acupuncture Point and Channel Energetics. München: Kiener; 2014

\section{Bibliografie}

AkupunkturPraxis 2020; 1: 33-45

DOI 10.1055/a-1110-5473

ISSN 1438-2563

(C) 2020. Thieme. All rights reserved.

Georg Thieme Verlag KG, Rüdigerstraße 14,

70469 Stuttgart, Germany 